DOI: https://doi.org/10.47405/mjssh.v6i11.1121

\begin{tabular}{|c|c|}
\hline 4 & Malaysian Journal of Social Sciences and Humanities (MJSSH) \\
\hline $\begin{array}{l}\text { Malaysian Juoural of } \\
\text { Social ccciecces and }\end{array}$ & Volume 6, Issue 11, November 2021 \\
\hline (MJ-sSH) & e-ISSN : 2504-8562 \\
\hline & $\begin{array}{l}\text { Journal home page: } \\
\text { www.msocialsciences.com }\end{array}$ \\
\hline
\end{tabular}

\title{
The Extrinsic Effects of Unauthenticated Publications on Social Media on Readers' Judgement and Perception
}

\author{
Janak Ashok Teckwani, Chew Siew Wei \\ ${ }^{1}$ Faculty of Social Sciences and Leisure Management, Taylor's University Lakeside Campus, Malaysia \\ Correspondence: Janak Ashok Teckwani (janakashokteckwani@sd.taylors.edu.my)
}

\begin{abstract}
The dissemination of unauthenticated publications on social media platforms has increased over the years and the factors, congruent to the extrinsic effects of these publications, are less studied and analyzed. It is important to understand the relationship between social media content deception and the sharing behavior of all the readers who engage with it on a daily basis. This research studies the perception of 16 Malaysian individuals on the antecedents of falling prey and sharing fake news as well as their perceptions on the general repercussions that are deemed prominent. Emphasizing on the prevalence of mass sharing, these publications continue to significantly impact readers with time, as exemplified in the findings of the research. Study findings also indicate that crucial periods, such as the COVID-19 pandemic, inculcate curiosity and vulnerability and thus making it easier for readers to fall prey to social media postings that serve as a facade for all misinformation and disinformation.
\end{abstract}

Keywords: fake news sharing, social media platforms, deception, COVID-19, misinformation

\section{Introduction}

In this day and age, we are all susceptible to digital divide and it is very imperative to understand how social media has a vital role in the life of daily readers. The impact it poses can be both positive and negative, depending on how it is utilized on a daily basis. As such, the proliferation of fake news and the spread of misinformation has not only brought menace, but to an extent, physical harm as well. It has also increased and brought about suffering to several readers, especially during crucial times when curiosity is at peak. An example would be how scammers jump to the opportunity of spreading misinformation and fake news to catapult their platforms into fame by multiplying the existing traction (Collins et al., 2020).

Keeping that in mind, readers consume information from distinct social media platforms almost every day and what is read can shape the mindset, depending on the context of the publication. Some of these platforms include Instagram, Twitter, Facebook and WhatsApp. On the whole, detecting fake publications on these platforms is a complex and challenging issue. Certain cues consolidate the perception of the readers, especially when they think it resembles authentic publications posted in official sites. There is a bigger price to pay when the traction is large, and this is because more people partake as well as conform with the general consensus while not paying heed to the facts displayed. When the publication and fake news appears real, it affects informational credibility and erodes readers' judgment. 
The effects are not just limited to online processes, but they also spill over into every other part of our lives. Research on the reasoning has also only focused on heuristic and peripheral cues that justify the rapid decisions that people make when sharing or spreading such publications (Buchanan, 2020). However, the factors that attracted them to these publications, while projecting a sense of reality to the readers, is where more research is required. This is also focused more on a societal level, contrary to most research which focuses on intrinsic analysis. With more people altering social media platform outlooks from time to time, it becomes more difficult to differentiate between what is real and what is fabricated. The addition of multiple sources puts on a facade, making people believe that the information shared is somewhat legitimate.

\section{Literature Review}

Concomitant to the idea that social media is one of the most effective and ineffective mediums of information sharing, unauthenticated publications have significantly affected people's trust (Cheng \& Chen, 2020). The information posted is also accessible in multiple platforms such as Facebook, Instagram, Twitter and the list goes on. The idea of mass sharing with the consensus of the general public, despite thorough checking on the authenticity of the information, forms a false perception which makes the publication. Such information is usually disseminated clandestinely on social media platforms as an effort to obfuscate the truth and eventually, manipulate public opinion (Vereshchaka et al., 2020).

The repercussions that usually transcend from these sharing is predominantly unpleasant, especially in crucial times where there is an existing influx of information. The speed at which information is shared on social media makes it virtually impossible for the process of determining informational credibility to occur at a rapid pace, therefore underscoring the exigency for of developing background checking systems to spot fake news (Tacchini et al., 2017).

An exemplified scenario would include how misinformation on the COVID-19 virus during the pandemic led to mass poisoning in Iran as well as mob attacks in India (Soltaninejad, 2020). There are also scammers who jump on the opportunity of selling "mineral miracle supplements" during the pandemic, when in reality these supplements are diluted bleach (Coleman, 2020). The predominantly discussed negative behavioural outcomes are shared in empirical studies based on examination of root content, sharing history analysis of the fake story and hyper partisan writing and political mobilization message analysis (Allcott and Gentzkow, 2017; Potthast et al., 2017).

Misinformation spread during crucial periods can perpetuate ambiguity, putting readers in a position to abide by both unnecessary and false details. Another example would include partisan media, where the information shared can be biased and questionable. This has a major effect on readers who perpetually engage and read partisan news sources on social media platforms (Tsfati et al., 2020). With the effort of multiplying traction, fake news that cater specifically for a specific brand, product or even a viewpoint, which may be false, can intentionally sway and mislead readers (Potthast et al., 2017).

Research on the general outlook of unauthenticated publications and the immediate effect it has on readers has been scarce. Although such, previous studies have shown that there is a tendency for such categorical news to perpetuate for a longer period of time especially when it "goes viral". This generally leads to people sharing repeated headlines than new ones, without reviewing the information appropriately (Effron \& Raj, 2019). As mentioned, the consensus of many has the power of manipulating judgement and forming a new meaning of news that has been published.

Emphasizing on the widespread of such occurrences, social media usage is deemed ubiquitous and the misinformation cuts across almost all age groups and social classes (Maitland \& Lynd, 2020). While acknowledging that sharing fake news is a global concern, research outcomes have shed minimal insight into the motives of the sharing. False updates and fabrication of information can alter what was once the perception of readers on a certain topic of discussion, thus affecting their judgement. 
Authentication of fake news before sharing also had no particular effect due to lack of religiosity and time (Talwar et al., 2020). A study indicated that there is a negative association between online trust and authenticating news before sharing, which highlights the negating social comparison (Talwar et al., 2019). Keeping this in mind, there is also a positive association between the fear of missing out and the intentional sharing of unauthenticated news. With the idea that there is some sort of information provided, especially with the outlook so similar to an authenticated publication, people refrain from double-checking while being a victim of deception.

Viral circulations and fabricated news have become a grave concern, overall, especially due to anonymity and geographical distance. Previous studies have also shown that the nature of social media encourages the behaviour of ignorantly sharing fake news to other readers due to its ease, and these readers could potentially forward it and cause a snowball effect (Albright, 2017). The underlying behaviour of fake news is still ambiguous while aspects including social media fatigue, coping strategies, information overload as well as fear of missing out continue to intensify (Tandon et al., 2021; Luqman et al., 2017; Malik et al., 2020). Indeed, studies have also pinpointed that, intrinsically, pre-existing beliefs account for judgement. The structuring of messages and media affordances on these social media platforms, however, play a vital role in the sharing of fake news on said social media platforms (Newhoff, 2018).

\section{Problem Statement}

It is imperative to understand that the issue at large scrutinizes on the misinterpretation of news and information on social media, and this leads to the general public experiencing distinct emotions from the many versions they are presented with. The prevalence of readers being deceived increases because those who encounter misinformation on said platforms tend to actively spread it further by actually engaging with it (Buchanan, 2020).

Without the authentication of the information provided, the readers are oblivious and they are subjected to a series of inappropriate judgements. Recognizing that social media is considered to be a very powerful tool, it could not only be used to spread illumination but illicit incessant fear-mongering. Misinterpretation can lead to unwanted actions that indirectly perpetuates the condition to worsen. Concomitantly, a study on the extrinsic effects of unauthenticated publications is vital, especially in crucial times where information is easily accessible on social media.

\section{Research Questions}

What are the factors that lead to unauthenticated publication sharing and deception based on a reader's perspective? What are the general repercussions of misinformation on readers' judgement and perception based on the postings of unauthenticated publications on social media platforms, especially with it being accessible on a daily basis?

\section{Objectives and Significance of Research}

The research aims to scrutinize on the extrinsic effects of unauthenticated publications posted on social media platforms on the manipulation of perception and judgement of the daily readers. Highlighting that scarce research has been conducted on the subject matter, it is essential to understand the contributing factors and its significance towards the general public, considering that these publications play a pivotal role in information sharing which happens daily. It is also to bring about awareness on the possible repercussions of spreading fake news and misinformation to the general public, to both publishers and readers who share. 
The research will provide a clearer view on the importance of informational credibility with regards to shared material on social media platforms. Understanding that readers engage with and absorb information from these publications, the research should aid in cultivating the ability to distinguish between authenticated and unauthenticated postings on the platform, while also understanding that there can be general repercussions for perpetually sharing such publications to other readers. This study will also project the perspective of readers, based on their first-hand experiences, while showing how significant it can be for the appropriate information to be delivered through.

This research will not just be able to benefit daily readers, but also social media platforms and admins that publish the news articles and information that have been compiled from various sources. It will aid in providing appropriate judgements during the compilation process, so that these parties will only publish information from verified resources. This research will also benefit these platforms by emphasizing on the importance of background checks before consolidating information online and posting it to the general public. Understanding that social media has blended in as a daily necessity, both readers and admins could benefit by understanding signs of fabrication and replication of news and the implication of sharing ignorantly.

\section{Methodology}

\section{Participants}

Appropriate chronological procedures were followed upon the recruitment of participants and the management of the research interviews. It was also ensured that the demographics tallied with the recruitment procedures and the participants included 16 Malaysian individuals between the age of 18 and 49, irrespective of gender and socioeconomic status. The participants, too, were daily readers and/or users on distinct social media platforms, and they have also engaged with the sharing of information online. The age group, 18-49, serves as an appropriate scope to study the population that collectively covers $75 \%$ and more of the active Internet users in Malaysia since 2020 and they serve as the most prominent sample of individuals that engage in information sharing and reading online (MCMC Internet User Survey, 2020). Should the individuals express uneasiness or sudden disinterest upon being approached, they are then excused based on the circumstances that they are subjected to. It has been understood that misinformation can cause emotional instability due to personal reasoning, depending on the individuals. Participants were also recruited from a number of social media platforms, namely Instagram, WhatsApp and Facebook. Prior to confirmation, they were also given the opportunity to clarify their doubts by asking questions regarding the research.

\section{Sampling Method}

Considering that the readers' judgement and perception has a rather subjective take to it, a semistructured interview, with the necessary questions, was decided to be used to tackle the research topic. The participants were chosen via a non-probability sampling technique which is purposive sampling, selective of the criteria that they are regular, day-to-day social media users. There were prior communications done to set the necessary appointment dates and detailing for the ease of the process.

\section{Instruments}

The semi-structured interview conducted was paired with probing to maximize data collection. The questions predominantly touched the surface of social media and its construct, fabricated news and the repercussions that surround the day-to-day postings. The application used, on the other hand, was listed in the recruitment sheet and it is emphasized that no additional applications were used to carry out the interviews, other than what has been explicitly stated.

\section{Procedure and Data Analysis}


Prior to the development of questions utilised in the interviewing process, research had been done on existing articles that correspond with the topic of the research. The journals that were congruent to the crux of the research were used as reference, building an informational understanding which was imperative for the participants. Both knowledge-based and open-ended questions were developed for the interview, entailing the perceptions and the opinions of all the participants on the extrinsic effects of unauthenticated publications. Participants were notified on their interview schedules prior to the session, with the Zoom link as an ease point for access. A copy of the developed questions is attached in the appendix of the report, as a question sheet, for reference (Appendix A). The interview sessions were held confidentially, lasting for the approximate time frame between 30 to 45 minutes. There were instances where some of the participants had technical issues during the session, especially with network connectivity, and this led to unscheduled breaks. However, the issues were dealt with promptly and the recording was kept pristine.

The recordings were then transcribed verbatim, without leaving out any contributing detail for the analysis. The quotes used during analysis were kept as is without paraphrasing, as an act to preserve the authenticity of acquired data. Prior to the commencement of the session, the participants were sent a digital copy of the informed consent entailing all the necessary information regarding the interview. They were also assured of anonymity and confidentiality; the participants were also well-informed about their rights during the session. They had the right to withdraw from the research at any particular point of the interview if it got overwhelming or if they were uncomfortable. One participant refrained from being video recorded, which was also a part of the entitled rights. The study, as a whole, received a valid approval from the Taylor's University Human Ethics Committee, Reference number: HEC 2021/033. A copy of the approval (Appendix H) and sample transcript (Appendix B) are attached, independently, in the appendix for necessary referencing. After tabulation, the participants were assured that it is mandatory for the recordings to be deleted in accordance with the agreement and information sheet provided. Post interview, the participants were debriefed and a service sheet containing a list of social services was provided (Appendix F and G). Upon tabulation, recurring patterns were derived for thematic analysis to occur according to the provided data.

A thematic analysis was conducted to examine data generated from the participants' responses to aid as a research tool for both psychological and social interpretation. The emerging themes were also identified with the use of an inductive approach and it was tallied with the research data. The process included scrutinizing and highlighting codes, similar patterns as well as differences to form potential themes that cater to the research question of the study. It was also emphasized that every interpretation was made based on the participants' narrative, and not in any way impacted by personal biases or grounding. Preliminary codes were produced to acquire the key points and common meanings, as well as the recurring patterns which reflects the significant reasoning participants used to rationalize their perspective and views. The method of coding was very inclusive in the sense that it encompasses quotes from the majority of the participants, regardless of the length. As part of the initiative to organize the potential themes, each of the specific themes were highlighted in the results and discussion section as per the interview excerpts.

\section{Results}

The themes that were identified while coding and analyzing the raw data obtained from the responses of 16 participants who partook in the scheduled interviews include Deceiving Outlook and Semantics Catapulting Fabrication, Mob Mentality Enhances Fallacy with Traction, Reluctance to Evaluate Sources for Informational Credibility, Pre-Existing Relationships and Mutual Trust in Sharing Publications and Ignorantly Disseminating False News Causing Repercussions. All of the stated themes collectively answer the research questions and it incorporates distinct points of view. Within these themes, there are specific quotes that complement the explanation, and said quotes serve as examples of the participants' perspectives. Understanding that interchangeable responses may be generalized as the majority, there are also outliers providing unorthodox perspectives on the discussed question. 


\section{Deceiving Outlook and Semantics Catapulting Fabrication}

Majority of the participants expressed a vital point on how prominent the outlook and semantics of unauthenticated publications are as a facade. What is read may attract public attention, especially when the descriptive details of the article posted is similar to a reviewed and authenticated one. Although such, these participants have mentioned that they have fallen prey to such publications due to the inability to distinguish a superficial article, especially when it mirrors the outlook of a reputable source. This leads to a reciprocation where they instantly disregard the need to further research and instead, share the news to the people they are close with as well as the general public. "Hogen", 23, brought to attention regarding the false use of official logos and the possibility of design replication which forms an illusory truth effect: "when it looks reputable it's because they use like the official logos, or the design, very closely replicate that off from the official source. So at first glance, you know, you will think that this unauthenticated publications are real."

Concomitantly, the usage of language in such publications would be able to denote the authenticity of news posted as official news incorporates the appropriate use of grammar and vocabulary. This is in regards to the review process that a manuscript usually goes through before it is published and posted in public domains. "Soho", 20, mentioned "But it's mainly on, as I said, it's mainly on like, how they put out news, right. So the first line is what makes it or breaks it.". It can definitely be clear-cut to people that an irregular sentence structure from the beginning of the article or news itself cancels the thought of it being authenticated. The procedure is usually thorough to maintain a certain standard, especially in journalism or any other official bodies per se.

However, "Sanju", 34, also projected an opinion on other distinct details that can contribute to the fallacy of readers' perception. She brought to light:

...in marketing you're writing in bolder, or, you know, in in capital, or in a colourful writings, you know, or big wordings to get your attention, definitely, if you look at a dull script against well-decorated script you tend to want to read well, decorative script...

This translates to the comparison between a dull script and a well-decorated script where font size, colouring, style, spacing and structure forms an identity for the publication as it is posted. It would most certainly ignite a queer feeling for the readers when the font and writing does not complement the intention of the news.

The overall outlook, which includes the design and font, as well as the language has an important significance when it comes to an authenticated publication. The initiative of replicating official logos, trademarks and any sort of identification could lead to the owners facing legal actions for violating copyright laws or infringement. Most of the participants have been deceived into thinking that the news posted is true, only to later realise that it almost exactly replicates the formatting of official pages.

\section{Mob Mentality Enhances Fallacy with Traction}

Eye-catching publications tend to gain traction at a quicker pace, no matter the authenticity. The participants shared their responses on the rationale behind mass sharing of fake news and misinformation which generally occur on a daily basis. While these sharing happen on a perpetual scale, it potentially forms an imagery in the mindset of daily readers that these publications are true. It, then, forms a snowball effect and influences a large number of those who encounter said publications on social media platforms, which include Instagram, Twitter, Facebook and so on. Large numbers translate into authenticity, and the chain reaction causes repercussions to the people involved in the sharing and those that they share it to. "Hogen", 23, expressed that when a number of individuals eventually invest themselves on one publication, they begin mass sharing, "..they tend to just want to believe one thing, and then they will just mass share on uh WhatsApp, especially through many of their different group...”. 
Mass sharing also strengthens the thought idea that if a large crowd shares the unauthenticated publication, it has the ability to make the content convincing as a whole. "Charlie", 24, puts this into perspective by sharing: "like when you see it news, and then you're like, oh, this sounded legit. Shared many times because news. These are very convincing.". People in power too are responsible in making the information convincing when it goes out to their subordinates, fans or the general pool of readers that they influence. There is a bond of mutual trust when these people share any information for that matter. Whether or not they tend to be accurate, there is a tendency for the readers to be convinced by the posted publication. "TJ", 22, emphasized on a premise which involves gaming streamers and the natural instinct to agree to superficial publications that revolve around them:

And there are some news articles that will post about streamers and certain stuff like that. And some are actually fake, but I would listen yeah, like mob mentality, that kind of thing. Like some would listen to it but would just, just.. just.. just believe in it. To put it simple, we worship the idols.

Another imperative observation is that negative publications actually evoke a stronger psychophysiological response than positive news. Such news dominates the headlines in today's world and it forms a negative bias where it affects how readers view current affairs. The existing fallacy is then enhanced as it reaches a large number of readers online. Bonny explains the increase in activity through such publication: "...but there they are the young people so before they verify the news, you know, looks interesting, negative attracts faster. So when you post stories, people respond to you, you'll get more in all this kind of engagement...". With accessibility almost anywhere, social media has developed new trends of fake news where there is a constant spreading of misinformation and this happens at a rapid scale.

Some of these publications are also malicious, depending on the severity and relevance to the situation. "Sanju", 34, explains how such information would be exploited in crucial times such as the COVID-19 pandemic, especially when there are many prying eyes:

I feel the main reason why people can be swayed by this information is because it's the current thing is the current trend. So you tend to believe like, for example, people say this virus can have this kind of effect, but it's not scientifically proven, you know, just people saying that it can have this kind of effect on the general fear and the general fear of the unknown, you know, that people have there is a swing, yeah, enough swing them towards believing this information.

In a sense, it acts as a perpetuating factor for people to cope with their fear of missing out on information by instantly sharing major headlines out of ignorance.

\section{Reluctance to Evaluate Sources for Informational Credibility}

Background checks have become a dilemma in this day and age, especially when readers are quick to share based on the headlines of publications that they encounter. They are quick to invalidate factual information as long as it becomes catchy enough to be shared and garner attention. The informational context may not appear as it seems, and similar to pseudoscience, it may seem legible due to the fact that it has been floating around on social media. "Quantico",21, shared his experience on public sharing and the desperation several people have to gain statistics:" You see them everywhere. But then then they are so desperate. Like they want to get views, they want to get clicks. Sometimes they don't even check your sources.".

There is an inherent sense of being complacent for some readers, even when they have the time to do the necessary checking regarding important publications. This leads to a rapid sharing of misinformation which can affect the perspective and judgement of other readers. They would react accordingly to fake news that has been shared, while completely disregarding the actual facts. "WeiWei", 21, briefly explained how readers would go over these publications after some time without questioning the details and context: 
So most of the time, I think that people won't actually go and check how legit is the news, but just sharing and maybe after a few hours when they scroll Facebook or anything. And they see some news that actually say that he was actually a fake news. And then they started sharing again, that is actually fake news."

There is, definitely, the possibility where a decent number of these readers do what is required before getting the information out on social media and other messaging platforms. They tend to ensure that this information is shared with the notion of being reasonable and accurate, according to the situation. "TJ", 22, highlights the general reaction that most readers would have upon reading an article that comes their way through her response:

Unless you really have the time in your hands, then only I think, people research on it. So I think in general, even if they have time on their hands, they're not going to research on it. They're not going to bother.

She mentioned how complacency has affected majority of the readers today and that they instantly absorb vague fake news and fabricated publications by concluding what the context would be from the headlines.

Although such, the readers who go the extra mile by researching the crucial details of a publication, before sharing it to their family members or friends, would still be considered minimal in number. A particular method includes scrutinising and cross-checking with other sources online to determine if the information is credible. If the article has been picked up by other official publishers or proven legitimate by the primary source, it would mean that the publication is authentic. "Hogen", 23 exemplified this when he explained that he does not instantly share news articles until it has been checked via a government website which aids in debunking fabrication to avoid readers from being misinformed:

So I remember that the government launched a website called 'sebenarnya.my'. And I found it to be really useful because they were quite up to date. And whenever I see a fake news, I will wait. And within 24 hours is normally reported, whether it's real or fake or not.

\section{Pre-Existing Relationships and Mutual Trust in Sharing Publications}

There is mutual trust between family members, friends and the people we surround ourselves with on a daily basis. The bond that is shared can be translated into comfort, where readers who receive fake news from these individuals, instantly buy into the idea that it is entirely authentic. What happens next is a set of events that contribute to the proliferation of unauthenticated publications in family and friendship groups, and gradually progressing to other communities that are filled with mutuals. On normal days, the frequency of these sharing can be concerning. "Quantico", 21 uses Facebook as an example, where people who generally know one another, come together to spread fabricated news and misinformation:

So about the specific parties I can see is like, not you know, people, they have this kind of like Facebook group, or they call it suisuichan, which is like, they go inside and blow water. So all these kind of groups will have stupid people who always post fake news. And it happens all the time.

These sharing typically occur with the press of one button and the readers who engage in said activity barely hesitate, especially after knowing that it is done to a pool of familiar individuals. "Chahi", 24 shares on how simple sharing fake news can be when it is in a group of people who can be considered trustworthy:

But it's obviously complete, you know, complete fabricated news. But for them, it's just that, oh, I'm just sharing the information forward. So you know, you're just 
using the forward button as in when you like, so $100 \%$ true, it's family members, friends.

Once the news has been shared and gone out of reach, the outcome is beyond the readers' control. It essentially garners more attention than required, only to later be debunked as a publication that has been wrongfully altered, fabricated or misguided.

Pre-existing relationships between friends and family promotes regular interactions, especially through messaging mediums and social media. It is commonly presumed as a method to keep in touch and the messages range from article forwards to daily headlines. "WeiWei", 21, opens up about how she has seen the postings in the family group:

...when everyone like stay at home, and they actually buy into the fake news, maybe because they really want to get engaged, like if they know something they want to share to their friends, their family members and such. So I think this is why they are easily trapped into fake news.

The messages, in general, aid in keeping the momentum going but they are done through inappropriate means and lack of knowledge on the discussed topic, depending on the article. The generation gap plays an imperative role in the effects of unauthenticated publications as well. It can be slightly more deceiving for the older generation, especially due to the digital divide. Younger generations are able to make regular background checks on the Internet after they have encountered a suspicious article, while some from the older generation struggle to do so. "Soho", 21, in essence, highlights the distinction as she observes the rate of sharing activity:

I have been in this particular WhatsApp group for the past, I think, six, seven years, more than seven years. And they are like aunties and uncles and close family members. And they have so much of news to share. And they are the ones that are like a lot more into the moment like a news get sent to them, or even my own, like, my own family members at the moment, and news gets sent to them.

This age factor is further cemented by a different participant, "TJ", 21, and the response was: "...yeah, that's why I said like, I think age plays a factor. I think it's more deceiving to someone who is older. Because they don't really do their research.".

\section{Ignorantly Disseminating False News Causing Repercussions}

Sharing publications ignorantly, without reviewing the authenticity, can bring about repercussions to new readers and the people they share it to. These repercussions are a reflection of how readers react when they encounter such publications at first sight. What is seen as a casual and rapid forward can be repeated perpetually with the other readers who get the article. Once it has gained public attention, it becomes significantly difficult for sources to retract these publications. Even with a cover up, the nuances will still remain as digital footprints on social media. Majority of the participants expressed how unauthenticated publications evoke a plethora of unpleasant responses, including anger and fear, as it has been shared to other readers. "Hogen", 23, brought to light how it affects readers, especially in crucial times such as the COVID-19 pandemic:

...because um, when they share things like that, it causes fear mongering. And like the most recent right now is due to COVID. And then you will be like, oh, this area had like this many cases, but then there were no cases there at all and so it just makes everyone paranoid.

It also becomes evident that people resort to unnecessary details that may be irrelevant situations. It could also sway many readers into practicing new coping mechanisms that was not scientifically reviewed or proven by the authority. "Garam", 34, shares how a member of his family reacts upon reading fake news and practices what has been dictated without questioning: 
So what happened is one of our unsuspecting elderly, so she's she sends all these articles. And apparently, in that article, you could use turmeric with warm water to kill the virus. So for example, like the one of the COVID-19 turmeric, that some of my relatives started drinking turmeric.

The outcomes are unpredictable but they can definitely be dire in certain scenarios, depending on the relevance of the topic. Readers who don't fact check before they decide on sharing articles, could potentially hurt the sentiments of other readers and communities. "Milo", 21, points out one prominent implication from the sharing of unauthenticated publications: "....they would actually have made a negative impact on like, they would give prejudices to other races and people that really thinks that the links are legit or anything...". Such occurrences may lead to serious altercations and verbal spats on social media, which could potentially worsen the existing setback. "TJ", 22, sheds light on the potential effect caused by an online clash, rooting a fabricated publication:

Uh basically, like I said, like if they were to share a news publication that is, that is actually fake, just fake news, there are arguments are bound to happen. Because one party will believe it and one party don't believe it. And that's where a war would start happening. kind of thing.

On the contrary, a participant, "Tave", 21, provided an unorthodox view on how sharing unauthenticated publications can be positive: "Well, in a positive way, my mom has a lot of topics to talk to her friends about.". This, indirectly, shows how healthy discussions over an article can determine its validity, through the exchange of views from various parties. It is beneficial to the community as readers abstain from sharing fake news immediately after encountering them and they are able to talk about it while learning facts on the subject matter.

\section{Discussion}

The inability to evaluate informational credibility on social media has affected a large number of readers and this potentially demeans their perceptions as well as judgements. There is a relative lack of filtering when it comes to posting information on social media platforms, including Facebook, Twitter and Instagram (Celliers \& Hattingh, 2020; Li \& Suh, 2015). Despite its importance, research on the subject matter has been minimal, especially focusing on the factors that lead to readers being deceived on a daily basis. Throughout the qualitative study, it has been revealed that readers who engage in the sharing of unauthenticated publications on social media share similar thoughts and perception on the extrinsic effects upon encountering these publications. Although such, it is important to note that the dramatic increase in frequency of sharing fake news during a crucial period, especially the COVID-19 pandemic, has been frighteningly significant and this affects the general information consumption that drives actions. With this as an example, the premise of uncertainty hikes the emotional load of readers to the point that it can be unpleasant.

Although readers falling prey to unauthenticated publications and subsequently sharing to other individuals were predominantly derived from the idea of hijacked thinking, it was interesting to see how participants tackle the issue while highlighting vulnerabilities in a social premise. The social component makes these unauthenticated publications lethal, in the sense that readers are susceptible to encountering people who share the similar thought process and they offer information which further cements their belief. It is, in a way, denoted as motivated reasoning where the participants shared how they tend to believe news articles that conforms with their general worldview (Thaler, 2020). After a thorough analysis, extrinsic effects of unauthenticated publications on readers' perception as expressed by the participants of the specified criteria, frequently cited Deceiving Outlook and Semantics Catapulting Fabrication, Mob Mentality Enhances Fallacy with Traction, Reluctance to Evaluate Sources for Informational Credibility, Pre-Existing Relationships and Mutual Trust in Sharing Publication and even Ignorantly Disseminating False News Causing Repercussions. 
Indeed, the results are congruent with previous research, especially on the premise of online news postings and the possible repercussions. The identified themes are congruent to Social Comparison Theory, Self-Determination Theory and Theory of Rational Choice. This is due to the constructs of social media fatigue and fear of missing out which leads to the prevalence of fake news sharing (Beyens et al., 2016; Cramer et al., 2016). These readers share out of the need for relatedness, similar to keeping up with a trend, and they may even share these publications easily if it has the tendency to evoke their preferred social outcome. Keeping that in mind, there have been advances that go concurrently with the evolution of the digital age, and one particular aid that the participants brought to light is the government-based website, 'sebenarnya.my'. This website is essentially responsible for verifying the authenticity of news that has been posted online (Daud \& Zulhuda, 2020). With the emphasized examples made by the participants, it confirms that the government portal has provided opportunities for readers to be more careful with what they imbibe online.

Unauthenticated publications posted on social media occasionally have an aesthetical resemblance to the legitimate and credible content posted as mainstream news. It attracts the attention of other readers, especially through similar logos, web design and standard fonts, which was reiterated by majority of the participants. Source features aid in the indication of authorship or verification, which can be relatively deceiving when it is projected on fake news (Molina et al., 2019). In relation to the general consensus, most of the participants relatively shared similar views on the outlook and semantics of such publications, and how they garner views as well. The reluctance to evaluate sources has brought about interesting inputs from the participants. The responses support the previous findings of readers sharing repeated headlines without appropriately reviewing the shared information.

Another point that the study denotes is the pre-existing relationships that catapult fake news sharing. There is a sense of mutual trust which makes regular sharing of unauthenticated publications somewhat believable. However, one participant responded uniquely, which went against the consensus, and it was regarding the possible discussions it can bring to the family. Discussions could aid in addressing the underlying facts or misinformation to avoid further sharing, which could cause a chain reaction. The responses acquired on the reluctance to evaluate sources complement previous studies, where there was a negative association between online trust and authentication of news prior to sharing.

Information consumers are easily swayed by traction and the responses on mob mentality, although prevalent, were interesting. While some found that mass sharing on its own has a significant impact, one participant brought to light regarding the role of people in power. Readers of a certain social group are generally susceptible to being influenced by the individuals they idolise or the authoritative figure. This contributes to the impact on the credibility of misinformation with the increase of online partisan media (Tsfati et al., 2020). The informational consumption becomes questionable, especially among the readers who are often exposed to partisan news sources. Research on people of influence who do endorsements on social media, and share information, has been minimal; it could potentially widen the perspective of daily readers to understand the limit of misinformation that is rooted from these "influencers" specifically.

On the topic of repercussions, ignorance can be detrimental to the readers that share and those who encounter the publications midway. Highlighting the fact that it can cause fear and other unpleasant responses, these unauthenticated publications are not limited to situational context. Fake news that are spread during crucial times, such as the COVID-19 pandemic, diminishes the idea of rational sharing, which in turn creates misinformation and disinformation. There is moral and intangible harm which makes readers get a hazy understanding on the actual details of the situation (Montesi, 2020). This is also reflected in several participants' point of view of the experiences falling prey to unauthenticated publications posted on social media platforms, especially when the situation persists and a lot of attention is being put on the subject matter.

\section{Implication and Recommendation for Future Research}

There is a general variation in the demographics of the sample, inclusive of the age groups and socioeconomic status. It makes the study, as a whole, a very enhanced premise with the combination in 
the sample being very representative and diverse. With regards to the research question, there were both positive and negative views on the repercussions of unauthenticated publications and how people are starting to react to them with time. Although such, this can be an interpretation of evolving perceptions especially with the increasing prevalence of repeated daily postings of unauthenticated publications on social media. The research would also aid by increasing awareness on the subject matter, for both existing and new readers. It was also very intriguing to see all the participants provide their input on a topic that has a major effect in our society today. The acquiescence is addressed on how people are swayed by fake and fabricated news as well as misinformation especially on a daily basis. There are serious victims of this deed which makes the topic taboo, no matter the platform of usage.

However, even with a representative sample, the topic can account for more subjective opinions and perceptions through a repeat study. Another limitation would be that the research is conducted on a wide online community and the reliance on secondary sources due to lack of research on the scope. Purposive sampling also challenges the optimal validity; thus, the criterion can be further narrowed down to exploring specific demographics. As for the recommendations, it would also be interesting to scrutinize from a victim's point of view and how these publications have lured and majorly affected them as the primary subject of discussion in these publications. Future studies should include said additional criteria for an extended analysis on extrinsic effects of unauthenticated publications from a victim's point-of-view. The study should also be repeated by experimenting on the ability to distinguish fake news on a specific social media platform before comparing it to other platforms.

\section{Conclusion}

This study sheds light on the extrinsic effects and social implications of unauthenticated publications on social media and how it affects readers' perceptions and judgements, especially being subjected to reading said platforms daily. This study has also highlighted the comparison of how readers are deceived by the general outlook of these publications as they further share it to other family members, friends, acquaintances and public readers in general. The large influx of information shared on social media platforms can hinder the judgement of readers and cause misconception, especially during crucial periods such as the COVID-19 pandemic. Moreover, the ability to distinguish between authenticated and unauthenticated publications requires emphasis on detail, specifically on similarities and differences. This can curb the ignorant sharing of articles under the basis of traction and implied mutual trust. Addressing the issue that fake news has a hand in the proliferation of misinformation, social media admins can contribute by engaging in thorough background checks to filter irrelevant details before it goes out to the general public. The study encourages a thought-provoking discussion between readers and the people they share publications which could aid in tackling the issue at large.

\section{References}

Albright, J. (2017). Welcome to the era of fake news. Media and Communication, 5(2), 87-89. https://doi.org/10.17645/mac.v5i2.977

Allcott, H., \& Gentzkow, M. (2017). Social media and fake news in the 2016 election. https://doi.org/10.3386/w23089

Barrett-Maitland, N., \& Lynch, J. (2020). Social media, ethics and the privacy paradox. Security and Privacy From a Legal, Ethical, and Technical Perspective. https://doi.org/10.5772/intechopen.90906

Beyens, I., Frison, E., \& Eggermont, S. (2016). "I don't want to miss a thing": Adolescents'fear of missing out and its relationship to adolescents' social needs, Facebook use, and Facebook related stress. Computers in Human Behavior, 64, 1-8. https://doi.org/10.1016/j.chb.2016.05.083

Buchanan, T. (2020). Why do people spread false information online? The effects of message and viewer characteristics on self-reported likelihood of sharing social media disinformation. PLOS ONE, 15(10), e0239666. https://doi.org/10.1371/journal.pone.0239666 
Celliers, M., \& Hattingh, M. (2020). A systematic review on fake news themes reported in literature. Lecture Notes in Computer Science, 223-234. https://doi.org/10.1007/978-3-030-45002-1 19

Cheng, Y., \& Chen, Z. F. (2020). The influence of presumed fake news influence: Examining public support for corporate corrective response, media literacy interventions, and governmental regulation. Mass Communication and Society, 23(5), 705-729. https://doi.org/10.1080/15205436.2020.1750656

Coleman, A. (2020, August 12). 'Hundreds dead' because of COVID-19 misinformation. BBC News. https://www.bbc.com/news/world-53755067

Collins, B., Hoang, D. T., Nguyen, N. T., \& Hwang, D. (2020). Trends in combating fake news on social media - a survey. Journal of Information and Telecommunication, 1-20. https://doi.org/10.1080/24751839.2020.1847379

Cramer, E. M., Song, H., \& Drent, A. M. (2016). Social comparison on Facebook: Motivation, affective consequences, self-esteem, and Facebook fatigue. Computers in Human Behavior, 64, 739-746. https://doi.org/10.1016/j.chb.2016.07.049

Daud, M., \& Zulhuda, S. (2020). Regulating the Spread of False Content Online in Malaysia: Issues, Challenges and the Way Forward. International Journal of Business and Society, 21, 32-48.

Effron, D. A., \& Raj, M. (2019). Misinformation and morality: Encountering fake-newsheadlines makes them seem less unethical to publish and share. Psychological Science, 31(1), 75-87. https://doi.org/10.1177/0956797619887896

Li, R., \& Suh, A. (2015). Factors influencing information credibility on social media platforms: Evidence from Facebook pages. Procedia Computer Science, 72, 314-328. https://doi.org/10.1016/j.procs.2015.12.146

Luqman, A., Cao, X., Ali, A., Masood, A., \& Yu, L. (2017). Empirical investigation ofFacebook discontinues usage intentions based on SOR paradigm. Computers in Human Behavior, 70, 544555. https://doi.org/10.1016/j.chb.2017.01.020

Malaysian Communications and Multimedia Commision. (2020). Internet Users Survey 2020. https://www.mcmc.gov.my/skmmgovmy/media/General/pdf/IUS-2020-Report.pdf

Malik, A., Dhir, A., Kaur, P., \& Johri, A. (2020). Correlates of social media fatigue and academic performance decrement. Information Technology \& People, 34(2), 557-580. https://doi.org/10.1108/itp-06-2019-0289

Molina, M. D., Sundar, S. S., Le, T., \& Lee, D. (2019). Fake news" is not simply false information: A concept explication and taxonomy of online content. American Behavioral Scientist, 65(2), 180212. https://doi.org/10.1177/0002764219878224

Montesi, M. (2020). Understanding fake news during the COVID-19 health crisis from the perspective of information behaviour: The case of Spain. Journal of Librarianship and Information Science, 096100062094965. https://doi.org/10.1177/0961000620949653

Newhoff, D. (2018). Why do we share fake news? The Illusion of More. https://illusionofmore.com/why-do-we-share-fake-news/

Potthast, M., Kiesel, J., Reinartz, K., Bevendorff, J., \& Stein, B. (2018). A Stylometric inquiry into Hyperpartisan and fake news. Proceedings of the 56th Annual Meeting of the Association for Computational Linguistics (Volume 1: Long Papers). https://doi.org/10.18653/v1/p18-1022

Soltaninejad, K. (2020). Methanol mass poisoning outbreak, a consequence of COVID-19 pandemic and misleading messages on social media. The International Journal of Occupational and Environmental Medicine, 11(3), 148-150. https://doi.org/10.34172/ijoem.2020.1983

Stewart, E. (2021). Detecting fake news: Two problems for content moderation. Philosophy \& Technology. https://doi.org/10.1007/s13347-021-00442-x

Talwar, S., Dhir, A., Kaur, P., Zafar, N., \& Alrasheedy, M. (2019). Why do people share fake news? Associations between the dark side of social media use and fake news sharing behavior. Journal of Retailing and Consumer Services, 51, 72-82. https://doi.org/10.1016/j.jretconser.2019.05.026

Talwar, S., Dhir, A., Singh, D., Virk, G. S., \& Salo, J. (2020). Sharing of fake news on social media: Application of the honeycomb framework and the third-person effect hypothesis. Journal of Retailing and Consumer Services, 57, 102197. https://doi.org/10.1016/j.jretconser.2020.102197

Tandon, A., Dhir, A., Talwar, S., Kaur, P., \& Mäntymäki, M. (2021). Dark consequences of social media-induced fear of missing out (FoMO): Social media stalking, comparisons, and fatigue. Technological Forecasting and Social Change, 171, 120931. https://doi.org/10.1016/j.techfore.2021.120931 
DOI: https://doi.org/10.47405/mjssh.v6i11.1121

Thaler, M. (2020). The "Fake news" effect: Experimentally identifying motivated reasoning using trust in news. AEA Randomized Controlled Trials. https://doi.org/10.1257/rct.4339-1.0

Tsfati, Y., Boomgaarden, H. G., Strömbäck, J., Vliegenthart, R., Damstra, A., \& Lindgren, E. (2020). Causes and consequences of mainstream media dissemination of fake news: Literature review and synthesis. Annals of the International Communication Association, 44(2), 157-173. https://doi.org/10.1080/23808985.2020.1759443

Vereshchaka, A., Cosimini, S., \& Dong, W. (2020). Analyzing and distinguishing fake and real news to mitigate the problem of disinformation. Computational and Mathematical Organization Theory, 26(3), 350-364. https://doi.org/10.1007/s10588-020-09307-8 